\title{
Empleo de componentes sanguíneos, fármacos y procedimientos para el tratamiento de la hemorragia en ambiente militar
}

\author{
Navarro Suay R. ${ }^{1 a}$, Povo Castilla J. ${ }^{2 b}$, de Prádena y Lobón JM..$^{3 b}$, Hernández Abadía de Barbará A. ${ }^{4 a}$, \\ Sáenz Casco L. ${ }^{5 a}$, Álvarez Herranz P. ${ }^{6 a}$
}

Sanid. mil. 2013; 69 (2): 87-93; ISSN: 1887-8571

\begin{abstract}
RESUMEN
El control de la hemorragia es uno de los pilares de la medicina militar. El shock hemorrágico es la primera causa tratable de muerte en combate y supone un reto dentro de la logística sanitaria. El objetivo de este estudio es analizar el concepto y fisiopatología de la hemorragia, describir los componentes sanguíneos (concentrado de hematíes, plaquetas, sangre completa, plasmaféresis y componentes sanguíneos congelados), fármacos (fibrinógeno, complejo protrombínico, factor VII activado recombinante y ácido tranexámico) y procedimientos (control de la hemorragia a nivel táctico, cirugía del control del daño, resucitación del control del daño y tromboelastometría) que se pueden emplear para el control de la hemorragia en ambiente militar.
\end{abstract}

PALABRAS CLAVE: Hemorragia, Combate, Logística sanitaria, Componentes sanguíneos.

Use of blood components, medications and procedures for the hemorrhage treatment in the military environment

SUMMARY: The bleeding control is one of the topics in military medicine. Hemorrhagic shock is the leading cause of preventable death in combat and is a challenge in medical logistics. The objective of this study is to analyze the concept and physiopathology of the hemorrhage, describing blood components (packed red blood cells, platelets, whole blood, blood components and frozen blood components), medications (fibrinogen, prothrombin complex, recombinant activated factor VII and tranexamic acid) and procedures (tactical hemorrhage control, damage control surgery, damage control resuscitation and thromboelastometry) that can be employed to control bleeding in the military environment.

KEY WORDS: Hemorrhage, Combat, Medical logistics, Blood components.

\section{INTRODUCCIÓN Y OBJETIVOS}

La medicina militar se sustenta en varios puntos de apoyo y sin lugar a dudas, el control del shock hemorrágico es uno de sus pilares fundamentales. La hemorragia es la primera causa tratable de muerte en combate, el control adecuado del sangrado se considera primordial para garantizar la supervivencia del combatiente y el apoyo terapéutico encaminado a controlar la pérdida sanguínea, supone todo un reto dentro la logística sanitaria militar. Por todos estos motivos, en zona de operaciones la denominada «hora de oro» se suele reducir a «los diez minutos de platino» ${ }^{1,2}$.

El objetivo del presente estudio es analizar los distintos componentes sanguíneos, fármacos y procedimientos empleados en la medicina militar.

${ }^{1}$ Cte. Médico. Servicio de Anestesiología y Reanimación.

${ }^{2}$ Col. Médico.

${ }^{3}$ Tcol. Farmacéutico.

${ }^{4}$ Tcol. Médico. Servicio de Telemedicina.

${ }^{5}$ Cte. Médico. Servicio de Medicina Intensiva.

${ }^{6}$ Cte. Farmacéutico. Servicio de Farmacia Hospitalaria.

a Hospital Central de la Defensa Gómez Ulla. Madrid. España.

${ }^{\mathrm{b}}$ Centro de Transfusión de las Fuerzas Armadas. Madrid. España.

Dirección para correspondencia: Ricardo Navarro Suay. Servicio de Anestesiología y Reanimación. Hospital Central de la Defensa Gómez Ulla. Glorieta del Ejército 1. 28047 Madrid. España.r_navarro_suay@yahoo.es

Recibido: 16 de octubre de 2012

Aceptado: 7 de febrero de 2013

\section{MATERIAL Y MÉTODOS}

Se desarrolló una búsqueda no sistemática de artículos en la base Pubmed/MEDLINE de 1970 a 2011, limitada al idioma inglés. Se seleccionaron los más relevantes, incluyendo originales y revisiones. Las palabras clave utilizadas fueron: traumatismo, hemorragia, coagulopatía, transfusión masiva, cirugía control del daño, resucitación control del daño, combate, militar, guerra.

\section{CONCEPTO Y FISIOPATOLOGÍA}

Existen múltiples definiciones de hemorragia masiva, pero en relación a la temporalidad y a la cuantificación consideramos hemorragia $\operatorname{masiva}^{3}$ (Tabla 1 ):

- Pérdida de la volemia total a lo largo de 24 horas, lo que supondría la reposición aproximada de 10 unidades de concentrados de hematíes para un paciente de $70 \mathrm{Kg}$ de peso.

- Pérdida del 50\% del volumen sanguíneo en unas 3 horas.

- Pérdida del volumen sanguíneo a un ritmo de $150 \mathrm{ml} / \mathrm{min}$.

- Tasa de pérdida de volumen sanguíneo de $1,5 \mathrm{ml} / \mathrm{Kg} / \mathrm{min}$ durante más de 20 minutos.

En el shock hemorrágico asociado al politraumatizado aparecen alteraciones del sistema hemostático que perpetúan, en muchas ocasiones, el sangrado. La coagulopatía dilucional se produce por dilución de los factores de coagulación y plaque- 
Tabla 1. Tipos de hemorragia: signos y síntomas. (Modificado de: American College of Surgeons Committee on Trauma: Advanced trauma life support for doctors (ATLS) student course manual, 8th edition. Chicago, IL: American College of Surgeons; 2008)

\begin{tabular}{|c|c|c|c|c|}
\hline & Clase I & Clase II & Clase III & Clase IV \\
\hline Pérdida sanguínea (\%) & $<15$ & $15-30$ & $30-40$ & $>40$ \\
\hline Pérdida sanguínea (ml) & 750 & $800-1500$ & $1500-2000$ & $>2000$ \\
\hline PAS & Sin cambio & Normal & Reducida & Muy baja \\
\hline PAD & Sin cambio & Aumentada & Reducida & Indetectable \\
\hline Pulso & Normal & $100-120$ & 120 (débil) & >120 (muy débil) \\
\hline Relleno capilar & Normal & Lento $(>2 \mathrm{~s})$ & Lento $(>2 \mathrm{~s})$ & Indetectable \\
\hline Respiración (/min) & Normal & Normal & Taquipnea $(>20)$ & Taquipnea $(>20)$ \\
\hline Diuresis (ml/h) & $>30$ & $20-30$ & $10-20$ & $0-10$ \\
\hline Extremidades & Normal & Pálidas & Pálidas & Pálidas y frías \\
\hline Estado mental & Alerta & Ansioso & $\begin{array}{l}\text { Ansioso / } \\
\text { Somnoliento }\end{array}$ & $\begin{array}{l}\text { Somnoliento / Confuso o } \\
\text { inconsciente }\end{array}$ \\
\hline
\end{tabular}

PAS = Presión arterial sistólica; PAD = Presión arterial diastólica.

tas, tras infusión de grandes volúmenes de diferentes fluidos. La hipotermia, que frecuentemente se observa en estos pacientes, disminuye la actividad enzimática de las proteínas plasmáticas y de la función plaquetaria. La acidosis producida, como resultado de una disminución de la perfusión y un aumento del metabolismo anaeróbico conduce a la acumulación de ácido láctico, afectando a la funcionalidad de las enzimas plasmáticas y plaquetas, así mismo, reduce la transformación de protrombina. El estado de hiperfibrinolisis es muy común en el politraumatizado. Hay que destacar la coagulopatía inducida por la anemia, ya que los hematíes intervienen de forma mecánica y funcional en la hemostasia primaria. Finalmente se ha observado la existencia de una coagulopatía de consumo, inducida por la exposición del Factor Tisular en el endotelio lesionado, que conduce a la activación de la coagulación y el consiguiente consumo de plaquetas y factores de coagulación ${ }^{4-6}$. El empleo de componentes sanguíneos, fármacos y procedimientos pueden atenuar estos procesos fisiopatológicos. (Tabla 2 y 3 )

\section{Componentes sanguíneos}

\section{a. Concentrado de hematíes ( $\mathrm{CH})$}

Los hematíes mejoran el transporte de oxígeno a los tejidos, contribuyen a la homeostasis gracias a su influencia bioquímica, funcional y por el efecto reológico sobre las plaquetas. También tienen un efecto procoagulante sobre la trombina ${ }^{7}$.

La transfusión de $\mathrm{CH}$ como terapia para la estabilización a nivel perioperatorio se considera primordial en los escalones quirúrgicos avanzados que despliegan en zona de operaciones ${ }^{8}$. Hay múltiples estudios que han analizado el empleo de $\mathrm{CH}$ en los diferentes conflictos durante el siglo $\mathrm{XX}^{9}$. Actualmente, en el ejército estadounidense se postula comenzar la resucitación de un paciente con hemorragia incontrolable administrando $\mathrm{CH}$ y si se requieren cuatro o más unidades, añadir plasma fresco congelado ${ }^{10}$.

\section{b. Plaquetas (P)}

La hemostasia primaria está caracterizada por la formación de un acúmulo de plaquetas. Este mecanismo es complejo, se necesita la presencia de fibrinógeno y la activación de los receptores glicoprotéicos localizados en la membrana de las plaquetas. La hemostasia se inicia tras la lesión de la pared vascular y la consiguiente adhesión de las plaquetas a nivel subendotelial. Posteriormente se activa el complejo GP IIb/IIIa que permite la agregación de más plaquetas ${ }^{6}$.

Desde que Miller ${ }^{11}$ tras su experiencia en la guerra de Vietnam, describe que la trombocitopenia secundaria a la hemodilución en pacientes con hemorragia masiva es la alteración más importante de la hemostasia, se ha intentado corregir este defecto. Entre 2004 y 2006, en un hospital militar estadounidense desplegado en Bagdag (Irak), se compararon 285 bajas a las que se les transfundió plaquetas con 149 bajas a las no. Ambos grupos presentaban hemorragia masiva. La supervivencia a las 48 horas $(82 \%$ vs $66 \%, \mathrm{p}<0,001)$ y a los 30 días $(62 \%$ vs $50 \%, \mathrm{p}=0,04)$ fue superior en el primer grupo respecto al segundo ${ }^{12}$.

\section{c. Sangre completa (SC)}

Tras la mejora en las técnicas de fraccionamiento sanguíneo, se consideró más eficiente el uso de $\mathrm{CH}$, PFC, crioprecipitados o concentrado de plaquetas, por separado. De esta forma, se limitaba el consumo de un recurso crítico y al mismo tiempo disminuía el riesgo de infección y de reacciones transfusionales en los receptores. Por estas razones, en la vida civil prácticamente se abandonó el empleo rutinario de $\mathrm{SC}^{13}$.

Sin embargo, debido a las peculiaridades del medio militar, en donde pueden coexistir dificultades en el aprovisionamiento, carestía para conseguir temperaturas óptimas, presencia de múltiples heridos con hemorragia masiva, en algunos ejércitos la utilización de SC podría ser una última y excepcional alternativa terapéutica. ${ }^{14,15}$. Las lecciones aprendidas en la guerra de Irak y Afganistán sobre el uso de SC, fueron recopiladas en la revisión de Spinella ${ }^{16}$. Entre el 2003 y el 2007 se transfundieron más de 6000 unidades de SC. Los resultados preliminares sobre 500 bajas con hemorragia masiva, muestran que el empleo de SC en estos pacientes es más eficaz que los $\mathrm{CH}$. El mismo autor, en un segundo estudio ${ }^{17}$ compara la supervivencia a las 24 horas y a los 30 días de un grupo al que se le transfundió $\mathrm{SC}+\mathrm{CH}+\mathrm{PFC}$ frente a otro tratado con $\mathrm{P}+\mathrm{CH}+\mathrm{PFC}$, encontrando mejores re- 
Tabla 2. Recomendaciones europeas del manejo de la hemorragia postraumática. (Modificado de Rossaint R, Bouillon B, Cerny V, Coats TJ, Duranteau J, Fernandez-Mondejar E, et al. Management of bleeding following major trauma: an update European guideline. Critical Care 2010, 14: R52.)

\begin{tabular}{|c|c|}
\hline Pautas de actuación ante la hemorragia masiva tras traumatismo & Evidencia \\
\hline Disminuir el tiempo entre el momento de la lesión y el inicio del control quirúrgico & $1 \mathrm{~A}$ \\
\hline Empleo prehospitalario del torniquete para control del sangrado en lesiones localizadas en extremidades & $1 \mathrm{C}$ \\
\hline Valoración del mecanismo lesional, región anatómica afectada, características del paciente & $1 \mathrm{C}$ \\
\hline Control inmediato de la hemorragia, una vez localizada la causa & 1B \\
\hline No se recomienda valoración del hematocrito como dato de laboratorio aislado & 1B \\
\hline Recomendación de valoración del Ácido Láctico en sangre y del Exceso de Base como monitorización & 1B \\
\hline Monitorización rutinaria con INR, TTPA, fibrinógeno y plaquetas & $2 \mathrm{C}$ \\
\hline Control precoz del sangrado abdominal empleando hemostáticos y/o packing & $1 \mathrm{C}$ \\
\hline Empleo de Damage Control Surgery en pacientes críticos con hemorragia y coagulopatía & $1 \mathrm{C}$ \\
\hline Recomendación de agentes hemostáticos tópicos en combinación con medidas quirúrgicas & $1 \mathrm{~B}$ \\
\hline Recomendación de Tensión Arterial Sistólica entre 100-80 mmHg & $1 \mathrm{C}$ \\
\hline Fluidoterpia: Cristaloides inicialmente/solución hipertónica inicial/coloides en paciente inestable & $1 \mathrm{~B} / 2 \mathrm{~B} / 2 \mathrm{C}$ \\
\hline Recomendación para mantenimiento de la normotermia & $1 \mathrm{C}$ \\
\hline Inicio precoz de monitorización de la coagulación & $1 \mathrm{C}$ \\
\hline Empleo de fibrinógeno y monitorización mediante tromboelastometría y laboratorio & $1 \mathrm{C} / 2 \mathrm{C}$ \\
\hline Administración de antifibrinolíticos & $2 \mathrm{C}$ \\
\hline Administración de rFVIIa tras todas las técnicas de control previas y empleo de componentes sanguíneos & $2 \mathrm{C}$ \\
\hline Administración de complejo protrombínico para revertir hemorragia secundaria a anticoagulantes & $1 \mathrm{~B}$ \\
\hline
\end{tabular}

Tabla 3. Dosis recomendadas ${ }^{7}$.

\begin{tabular}{|c|c|}
\hline Producto & Dosis recomendadas \\
\hline Fibrinógeno & 3-4 g iv (Diluir con $50 \mathrm{ml}$ de agua para inyección durante 5-10 min y administración muy lenta sin superar los $5 \mathrm{ml} / \mathrm{min}$ ) \\
\hline Complejo protrombínico & Dosis individualizada dependiendo de cada fármaco $($ Octaplex $®$, Beriplex $®$, Protomplex $® \ldots)$ \\
\hline rFVIIa & $100 \mu \mathrm{g} / \mathrm{Kg}$ i.v \\
\hline Ácido Tranexámico & $10 \mathrm{mg} / \mathrm{kg}$ iv en bolo seguido $1 \mathrm{mg} / \mathrm{Kg} / \mathrm{h}$ i.v \\
\hline Hemoglobina & Dosis necesaria para mantenter $\mathrm{Hgb} 7-9 \mathrm{~g} / \mathrm{dl}$ \\
\hline PFC & $10-15 \mathrm{ml} / \mathrm{Kg}$ \\
\hline Plaquetas & Dosis necesaria para mantenter plaquetas $>50.0000$ \\
\hline
\end{tabular}

sultado en el primero. Por todo lo anterior, este autor considera que la SC es conveniente, segura y efectiva en ciertas situaciones militares $^{18}$.

\section{d. Plasmaféresis}

La aféresis es el procedimiento mediante el cual se separan los diversos componentes de la sangre, permitiendo el almacenamiento de plasma y plaquetas y devolviendo al donante los glóbulos rojos ${ }^{19}$.

Las sanidades militares estadounidense ${ }^{9}$ y británica ${ }^{20}$ cuentan con dispositivos de plasmaféresis en los hospitales de campaña. El ejército norteamericano comenzó a plantear esta alternativa a mediados de la década de los años 90 apostando por un diseño ligero, resistente y fácil de transportar. Finalmente, en 2004 se consiguió desplegar la primera máquina de aféresis en un hospital de campaña. Esta innovación provocó que en esa instalación sanitaria, disminuyese la transfusión de SC y se comenzara la terapia con los distintos hemoderivados ${ }^{14}$. Por su parte, el ejército británico logró disponer del primer dispositivo de aféresis en 2008, sin embargo hasta la fecha, sigue prevaleciendo el envío de plaquetas por vía aérea ${ }^{21}$, manteniéndose la plasmaféresis como método alternativo de segunda línea ${ }^{22}$.

\section{e. Componentes sanguíneos congelados ( $C H$ congelados, PFC, PC, Crioprecipitado)}

Los componentes sanguíneos congelados se pueden almacenar en los hospitales militares desplegados en zona de operaciones de una forma fácil, eficaz y segura, disminuyendo los requerimientos desde el territorio nacional, ajustando los niveles a la demanda real y reduciendo al mínimo su pérdida por vencimiento en la fecha de caducidad. Este beneficio es patente sobre todo con las plaquetas frescas, debido a que su vida media es de 5 días. El que algunas fuerzas armadas cuenten con plaquetas congeladas, ha supuesto una mejora considerable en el tratamiento integral de la baja y una gran ventaja logística, ya que su vida media alcanza un año ${ }^{23}$. Del mismo modo, el concentrado de hematíes congelado alarga notablemente su vida media, al pasar de 42 días a 10 años (si se mantiene a $\left.<60^{\circ}\right)^{24}$. El plasma fresco congelado se puede almacenar hasta 1 o 2 años a una temperatura de $<25^{\circ} \mathrm{C}^{23}$, pudiéndose emplear en aquellas situaciones donde la baja esté baja de factores de coagulación ${ }^{10}$.

El ejército holandés es pionero en la utilización de componentes sanguíneos congelados en zona de operaciones. Desde el año 2002, emplea su «banco de productos congelados» (incluyendo plaquetas) en Afganistán. Fruto de esta buena experiencia con los derivados congelados, este ejército está abandonando la 
utilización de sangre procedente de voluntarios donantes por el peligro de transmisión de enfermedades endémicas adquiridas en las zonas de despliegue como el paludismo o por la desventaja táctica que supone que estos militares se sometan a una extracción de sangre (por ejemplo, un soldado de infantería probablemente no estaría preparado para el combate hasta que no transcurrieran 24 horas de la extracción) ${ }^{24}$. La sanidad militar australiana también está empleando este sistema en Afganistán. En un estudio prospectivo, se reclutaron 158 pacientes intervenidos quirúrgicamente en 6 meses. De ellos 17 recibieron derivados plasmáticos congelados $(132 \mathrm{CH}, 75 \mathrm{PFC}$ y 22 unidades de plaquetas) ${ }^{25}$.

El crioprecipitado se obtiene centrifugando el PFC tras su descongelación entre 1 y $6{ }^{\circ} \mathrm{C}$. Contiene un $50 \%$ de factor VIII, un $20-40 \%$ de fibrinógeno y un $30 \%$ del factor XIII que estaban presente originalmente en el $\mathrm{PFC}^{26}$. Actualmente el aporte de los factores de coagulación presentes en los crioprecipitados, debe ser realizado mediante productos concentrados provenientes de la industria farmacéutica sometidos a inactivación viral. Por lo tanto la utilización de los crioprecipitados será en ausencia de disponibilidad de dichos concentrados.

En un estudio retrospectivo realizado en 2006 sobre bajas en combate con lesión vascular ${ }^{27}$, se compara un grupo al que se le trata con una terapia de resucitación (incluido crioprecipitado) con otro grupo al que no se le administra dicho tratamiento. A los dos grupos se les somete a una intervención quirúrgica. Este trabajo concluye que el empleo con terapia de resucitación obtiene una recuperación fisiológica más rápida que el grupo que no ha sido tratada con esa medida.

\section{Fármacos}

\section{a. Fibrinógeno}

Uno de los primeros requisitos para la formación del coágulo, es la conversión de una suficiente cantidad de fibrinógeno a fibrina. El fibrinógeno es el primer componente hemostático que disminuye debido a su consumo en el trauma y a la hemodilución tras la administración de fluidos durante la reanimación ${ }^{28}$. Por este motivo, se considera importante mantener unos niveles adecuados de fibrinógeno en el sangrado masivo ${ }^{15}$.

La serie recogida por Stinger et $\mathrm{al}^{29}$, demuestra por primera vez la asociación entre administrar cantidades elevadas de fibrinógeno y la mejora de la supervivencia en bajas de combate que han requerido transfusión masiva.

\section{b. Complejo protrombínico}

Los complejos protrombínicos contienen los factores II, VII, IX y X, que se consideran esenciales para la formación de trombina. Está indicado para revertir los efectos de los anticoagulantes orales y como tratamiento de alteraciones congénitas de la coagulación ${ }^{30}$.

En el artículo de Fries y colaboradores ${ }^{31}$ se defiende el empleo de complejo protrombínico como tratamiento efectivo para corregir la coagulopatía derivada del traumatismo, aun- que se necesitan más estudios científicos para confirmar esta indicación.

\section{c. Factor VII activado recombinante (rFVIIa)}

El empleo de rFVIIa está indicado en pacientes con hemofilia, déficit del FVII o trombastenia de Glanzmann. Su mecanismo de acción se basa en la activación del FXa, actuando sobre el factor tisular y en la superficie de las plaquetas $^{32}$. La mejora de la hemostasia en el punto de lesión es la base de su empleo como rescate en el traumatizado ${ }^{33}$. La primera vez que se documentó su utilización en este campo fue en 1999, cuando se administró a un soldado que presentaba lesión por arma de fuego en la vena cava inferior y había desarrollado coagulopatía ${ }^{34}$.

Varios estudios describen el empleo del fármaco como tratamiento de segunda línea en ambiente militar. Perkins et $\mathrm{al}^{35}$, analizó 117 pacientes tratados con rFVIIa en hospitales de campaña desplegados en Irak, y concluyó que la administración precoz de rFVIIa disminuyó la necesidad de $\mathrm{CH}$ en el $20 \%$ de los pacientes. También fueron recogidos resultados positivos en el trabajo de Spinella et $\mathrm{al}^{36}$, que describe un descenso de mortalidad a los 30 días sin encontrar un aumento de fenómenos trombóticos en aquellos pacientes con hemorragia masiva a los que habían administrado este medicamento. Por su parte Boffard ${ }^{37}$, encuentra que los pacientes con traumatismo no penetrante necesitan menos requerimientos transfusionales y la incidencia de síndrome de distrés respiratorio del adulto es menor. Sin embargo, una revisión de la Cochrane ${ }^{38}$ de 2007 concluye que la efectividad del rFVIIa como hemostático no está probada. Davies et al ${ }^{39}$ siguiendo las directrices inglesas ${ }^{40}$ analiza el momento de administración del rFVIIa en 95 pacientes atendidos por el ejército británico en Afganistán, encontrando que en el 92\% de los casos se habían seguido las instrucciones acordadas. Aunque estos estudios son prometedores, es necesaria una mayor experiencia para su empleo sistematizado en ambiente militar.

\section{d. Ácido tranexámico (ATX)}

La hiperfibrinólisis juega un papel trascendental en la fisiopatología del trauma ${ }^{41}$, por tanto los antifibrinolíticos pueden ayudar a reducir la pérdida sanguínea y su uso precoz puede contribuir a un mejor tratamiento de la coagulopatía traumática aguda ${ }^{15}$. El estudio aleatorizado $\mathrm{CRASH} 2^{42}$ encuentra que el ATX reduce en $0,8 \%$ el riesgo de muerte por sangrado en el paciente politraumatizado.

En un trabajo realizado Morrison et $\mathrm{al}^{43}$ en 2011 , se analiza retrospectivamente el papel del ATX en 293 bajas atendidas en instalaciones británicas y estadounidenses desplegadas en Afganistán. Los autores concluyen que el empleo de ATX mejora la coagulopatía y la supervivencia, siendo este beneficio mayor en aquellos pacientes que requieren transfusión masiva, y recomienda que este fármaco forme parte de los protocolos de tratamiento de la hemorragia en ambiente militar. En esta misma línea de actuación, en las guías de resucitación en combate escritas por Dawes et al ${ }^{44}$, se aconseja el empleo de ATX en las primeras 3 horas y repetir su ad- 
Tabla 4. Actuación en ambiente prehospitalario frente a hemorragia masiva, tipos de vendaje compresivo, torniquetes y productos hemostáticos.

\begin{tabular}{|c|c|c|}
\hline LESIÓN & ACTUACIÓN & SI CONTINÚA LA HEMORRAGIA \\
\hline Hemorragia catastrófica en cabeza, cuello, tórax & Vendaje + compresión & Aplicar los productos hemostáticos \\
\hline $\begin{array}{l}\text { Hemorragia catastrófica en miembros superiores } \\
\text { e inferiores }\end{array}$ & Torniquete & \\
\hline CATEGORÍA & PRODUCTO & AGENTE ACTIVO \\
\hline Vendaje compresivo & Emergency bandag & \\
\hline \multirow[t]{5}{*}{ Torniquete } & Combat aplication tourniquet (CAT) & \\
\hline & Mechanical advanced tourniquet (MAT) & \\
\hline & Autotransfusion tourniquet (A-TT) & \\
\hline & Emergency\&Military tourniquet (EMT) & \\
\hline & Integrated tourniquet system (ITS) & \\
\hline \multirow[t]{4}{*}{ Concentración de los factores de coagulación } & Quit Clot ${ }^{\circledR}$ & Zeolita \\
\hline & Quit Clot ACSTM ${ }^{\mathrm{TM}}$ & \\
\hline & TraumaDex ${ }^{\mathrm{TM}}$ & Polisacárido \\
\hline & WoundStat ${ }^{\mathrm{TM}}$ & Silicato de Aluminio, Ácido Poliacrílico \\
\hline \multirow[t]{6}{*}{ Agentes adhesivos } & Hemcom $®$ & Chitosan \\
\hline & Celox $^{\mathrm{TM}}$ & \\
\hline & TraumaStat & \\
\hline & $\mathrm{RDH}$ & Poly-N-Acetil Glucosamina \\
\hline & $\mathrm{mRDH}$ & \\
\hline & Super QR & $\mathrm{K}, \mathrm{Fe}$, Polimero hidrofílico \\
\hline \multirow[t]{3}{*}{ Factores procoagulantes } & Dry Fibrin Sealant & Trombina, Fibrinógeno, Ca, FXIII \\
\hline & Tachocomb H® & Colágeno, Trombina, Fibrinógeno \\
\hline & Combat Gauze & Caolín \\
\hline
\end{tabular}

ministración si se evidencia la presencia de fibrinolisis en la tromboelastometría.

\section{Procedimientos}

\section{Control de la hemorragia a nivel táctico}

TCCC (Tactical Combat Casualty Care) $)^{45}$, BATLS (Battlefield Advanced Trauma Life Support) ${ }^{46}$, o SVACOM (Soporte Vital Avanzado en Combate ${ }^{47}$ son algoritmos basados en recomendaciones militares estadounidenses, británicas o españolas respectivamente, que resaltan la importancia del tratamiento inmediato de la hemorragia en el combatiente, priorizando el control del sangrado $(<\mathrm{C}>\mathrm{ABC})$ sobre el aislamiento de la vía aérea y el control cervical (A) o el mantenimiento de la ventilación $(\mathrm{B})^{48}$. Para alcanzar este objetivo se emplean torniquetes ${ }^{49}$, ${ }^{50} \mathrm{y}$ vendajes compresivos ${ }^{51}$. Se han desarrollado productos hemostáticos de administración tópica ${ }^{52}$ y se aboga por el empleo alternativo de dispositivos intraóseos para la transfusión de hemoderivados $^{53}$. En algunos helicópteros de aeroevacuación médica se dispone ya de $\mathrm{CH}$, PFC y ATX para acortar los tiempos entre el momento de la lesión y el inicio de la hemoterapia ${ }^{22-54}$.
Los recientes conflictos de Irak y Afganistán, han renovado el interés en el desarrollo de nuevos y mejores agentes hemostáticos para su empleo en operaciones militares. Dependiendo de su mecanismo de acción principal, los podemos agrupar en: los que concentran factores de coagulación, los que actúan como adhesivos y los que incrementan la actividad procoagulante ${ }^{55}$ (tabla 4).

\section{Cirugía del control del daño (CCD)}

La CCD procede de un concepto naval definido como «capacidad de un buque para absorber el daño manteniendo íntegra su capacidad para cumplir la misión». Del mismo modo que tras sufrir un ataque los marineros se afanan rápidamente en apagar el fuego y cerrar las vías de agua, los cirujanos deben cohibir la hemorragia y preservar el contenido abdominal tan pronto como sea posible ${ }^{56}$. La principal diferencia respecto al ámbito civil radica en la gran cantidad de escalones logísticos y evacuaciones que soporta una baja, desde que sufre la lesión en el campo de batalla hasta que recibe el tratamiento definitivo en un hospital de territorio nacional ${ }^{57}$.

Según normativa OTAN ${ }^{2}, 58$, tras la estabilización inicial del herido, se procede a la realización de una intervención 
quirúrgica «abreviada». A continuación, se le ingresa en la unidad de cuidados críticos, para más tarde proceder a la evacuación a una instalación sanitaria de referencia. Allí se le someterá a una segunda intervención y por fin tras conseguir la estabilización, se trasladará de nuevo a la baja hasta el territorio nacional ${ }^{59-60}$.

\section{Resucitación del control del daño (RCD)}

Como es lógico, la RCD está íntimamente relacionada con el apartado anterior. Su objetivo principal es prevenir el desarrollo de la «triada mortal» (coagulopatía, acidosis e hipotermia) en el herido ${ }^{61}$. Este esfuerzo está encaminado a que el paciente sea estabilizado y abandone el quirófano con la mejor situación fisiológica posible. La RCD está basada en tres conceptos: hipotensión permisiva, disminución de aporte de cristaloides y aplicación de un protocolo de administración de hemoderivados que consigan una composición similar a la sangre completa ${ }^{62}$.

Niles y colaboradores ${ }^{63}$ publicaron un artículo sobre 347 bajas en combate atendidas en un hospital militar estadounidense en Irak durante los años 2003-2004. Todas ellas habían sido transfundidas (en el $84 \%$ únicamente con $\mathrm{CH}$ ), encontrando que el $38 \%$ presentaban coagulopatía (INR $\geq 1,5$ ). Consideraron este hecho como factor individual de riesgo de muerte.

Posiblemente, la hipotermia es más complicada de tratar en ambiente militar que en la vida civil. La evacuación de la baja puede sufrir retrasos por motivos tácticos, en helicóptero la temperatura ambiental va a descender y en caso de vuelo con cabina abierta el viento incrementará el efecto térmico ${ }^{64}$. Debido a que la presencia de hipotermia en los heridos en combate se asocia con incremento en la mortalidad, en 2006 se establecieron unas guías de prevención de la hipotermia en ambiente militar. Éstas, recientemente han sido analizadas en un estudio multicéntrico ${ }^{65}$ que concluye que desde que se instauró este protocolo, la incidencia de hipotermia en las bajas ha disminuido de manera significativa. Los estudios encaminados a prevenir la hipotermia en combate prosiguen, intentando encontrar dispositivos adecuados para este fin (mantas térmicas, calentadores de fluidoterapia...) ${ }^{66,67}$.

La presencia de acidosis en la baja de combate es bien conocida $^{68}$. En pacientes sometidos a una politransfusión, la acidosis normalmente está inducida por la hipoperfusión y por el exceso del ión cloro normalmente producido por la administración de corticoides. Conforme desciende el valor del $\mathrm{pH}$, las plaquetas adoptan una configuración diferente, se reduce la actividad de los factores de coagulación sobre la membrana celular de las mismas, el fibrinógeno se degrada y la formación de trombina disminuye ${ }^{15}$.

\section{Tromboelastometría (TE)}

La TE es una prueba que muestra las propiedades viscoelásticas de la sangre desde la formación del coágulo hasta su fibrinólisis, evalúa la función plaquetaria y se correlaciona con el proceso fisiológico de la hemostasia de una forma rápida. Puede ser una herramienta de monitorización farmacoterapéutica de hemoderivados así como de utilidad diagnóstica en el paciente en estado crítico, especialmente en aquellas situaciones que cursan con trastornos en la coagulación ${ }^{24-69}$. En 2008, un estudio del departamento de sanidad británico concluyó, que el uso del TE para la toma de decisiones, reducía la necesidad de transfusiones, disminuye los requerimientos de hemoderivados, mejorando la relación coste/beneficio y consigue un mejor control de los posibles efectos adversos secundarios a la administración de fibrinógeno y complejo protrombínico ${ }^{70}$.

En la actualidad, la sanidad militar alemana, británica y estadounidense cuentan con dispositivos ROTEM ${ }^{\circledR}$ (Rotational thromboelastometry, Pentapharm $\mathrm{GmbH}$ ) en sus instalaciones sanitarias de campaña desplegadas en Afganistán ${ }^{22}$. Las fuerzas armadas francesas contemplan el uso de esta técnica como fundamental en la monitorización de la hemorragia masiva ${ }^{71}$. El empleo de TE en ambiente militar ha sido recientemente descrito en un estudio que concluye que esta prueba detecta más anomalías en la coagulación que los test de TP y TTPA, puede ser una herramienta valiosa en el diagnóstico y tratamiento de la hemorragia masiva y puede predecir a los 5 min del test (A5) un estado de hipocoagulabilidad con sensibilidad/especificidad de $0,98 / 0,69^{72}$.

\section{CONCLUSIÓN}

El control de la hemorragia en la baja de combate es primordial. Es necesario conocer los diferentes componentes sanguíneos, fármacos y procedimientos más recientes para mejorar la atención de la baja en zona de operaciones y en territorio nacional.

Las diferentes lecciones aprendidas en los últimos conflictos bélicos orientan hacia un empleo precoz de concentrados de hematíes y plaquetas, la valoración de la sangre completa como método integral para el tratamiento del shock hemorrágico, disponer de componentes sanguíneos a través de aféresis o conservados gracias a la congelación, la utilización de fibrinógeno, complejo protrombínico, factor VII activado recombinante y ácido tranexámico como parte fundamental en el control de la coagulopatía, todo ello integrado en diferentes procedimientos que se inician a nivel táctico y que continúan en las instalaciones sanitarias (cirugía y resucitación del control del daño) y durante la evacuación de la baja.

\section{BIBLIOGRAFÍA}

1. Condon-Rall ME. A brief history of military anesthesia. En: Zajtchuk R, Grande CM. Anesthesia and perioperative care of the combat casualty. Washington: Textbook of military medicine; 1995. p. 855-96.

2. MC 0326/3 NATO Principles and Policies of Medical Support.

3. Castellanos A. Shock hemorrágico: evaluación y tratamiento inicial. En: Buisán F, Herrero E, Ruiz N, Páez M. Manual de cuidados críticos postquirúrgicos. Madrid: Arán; 2006. p.173-80.

4. Griffee MJ, DeLoughery TG, Thorborg PA. Coagulation management in massive bleeding. Curr Opin Anaesthesiol 2010; 23 (2):263-8.

5. Greer SE, Rhynhart KK, Gupta R, Corwin HL. New developments in massive transfusion in trauma. Curr Opin Anaesthesiol 2010; 23(2): 246-50.

6. Hardy JF, Moerloose P, Samama CM. Massive transfusion and coagulopathy: pathophysiology and implications for clinical management. Can J Anesth 2006; 53(6): S40-58. 
7. Rossaint R, Bouillon B, Cerny V, Coats TJ, Duranteau J, Fernandez-Mondejar E, et al. Management of bleeding following major trauma: an update European guideline. Critical Care 2010, 14:R52.

8. Ellison N. Transfusion therapy. En: Zajtchuk R, Grande CM. Anesthesia and perioperative care of the combat casualty. Washington: Textbook of military medicine; 1995. p. 321-39.

9. Bolan CD, Webster NR. Military transfusion practice. En: Zajtchuk R, Grande CM. Anesthesia and perioperative care of the combat casualty. Washington: Textbook of military medicine; 1995. p. 341-73.

10. Shawn C, Lounsbury D, Hertz S. Acute resuscitation and critical care. En Shawn C, Lounsbury D, Hertz S. War Surgery in Afganistan and Iraq. $1^{\text {a }}$ ed. Washington DC, Borden Institute, Walter Reed Army Medical Center; 2008.p. 17-52.

11. Miller RD, Robbins TO, Tong MJ, Barton SL. Coagulation defects associated with massive blood transfusions. Ann Surg 1971; 174: 794-801.

12. Borgman MA, et al. The ratio of blood products transfused affects mortality in patients receiving massive transfusions at a combat support hospital. $\mathrm{J}$ Trauma. 2007; 63:805-13.

13. Johansson PI, Ostrowski SR, Secher NH. Management of major blood loss: an update. Acta Anaesthesiol Scand 2010; 54 (9): 1039-49.

14. Repine TB, Perkins JG, Kauvar DS, Blackborne L. The use of fresh whole blood in massive transfusion. J Trauma 2006; 60 (6): S59-69.

15. Hrezo RJ, Clark J. The walking blood bank: an alternative blood supply in military mass casualties. Disaster Manag response 2003; 1(1):19-22.

16. Spinella PC. Warm fresh whole blood transfusion for severe hemorrhage: U.S. military and potential civilian applications. Crit Care Med 2008; 36(7) S340-5.

17. Spinella PC, Perkins JG, Grathwohl KW, Beekley AC, Holcomb JB. Warm fresh whole blood is independently associated with improved survival for patients with combat-related traumatic injuries. J Trauma 2009; 66(4):S69-76.

18. Kauvar DS, Holcomb JB, Norris GC, Hess JR. Fresh whole blood transfusion: a controversial military practice. J Trauma 2006; 61(1):181-4.

19. Perkins JG, Cap AP, Spinella PC, Blackbourne LH, Grathwohl KW, Repine $\mathrm{TB}$, et al. An evaluation of the impact of apheresis platelets used in the setting of massively transfused trauma patients. J Trauma 2009; 66(4):S77-84.

20. Doughty A. Mission impossible? Blood for the battle space. WMMMG. Queen Elisabeth Hospital, Birmingham 13 Oct 2011.

21. Mahoney PF. Images of anaesthesia. JR Army Med Corps. 2010; 156(4): 407-12.

22. Doughty HA, Woolley T, Thomas GOR. Massive transfusion. JR Army Med Corps. 2011; 157(3): 277-83.

23. Expósito JL. Oro rojo para las operaciones. Revista Española de Defensa. 2009; 250: 24-7.

24. Lelkens CC, Koning JG, Kort B, Floot IB, Noorman F. Experiences with frozen blood products in the Netherlands military. Transfus Apher Sci 2006; 34 (3):289-98.

25. Neuhaus SJ, Wishaw K, Lelkens C. Australian experience with frozen products on military operations. MJA 2010; 192 (4): 203-5.

26. Norda R, Tynell E, Akenblom O. Cumulative risks of early fresh frozen plasma, cryoprecipitate and platelet transfusión in Europe. J Trauma 2006; 60 (6): 41-5.

27. Fox CJ, Gillespie DL, Cox ED, Mehta SG, Kragh JF, Salina J, Holcomb JB. The effectiveness of a damage control resuscitation strategy for vascular injury in a combat support hospital: results of a case control study. J Trauma 2008; 64(2):99-106.

28. Hiippala ST, Myllyla GJ, Vahtera EM. Hemostatic factors and replacement of major blood loss with plasma-poor red cell concentrates. Anesth Analg 1995; 81 (2): 360-5.

29. Stinger HK, Spinella PC, Perkins JG, Grathwohl KW, Salinas J, Martini WZ. The ratio of fibrinogen to red cells transfused affects survival in casualties receiving massive transfusions at an Army Combat Support Hospital. J Trauma 2008; 64 (2): S79-5.

30. Samama CM. Prothrombin complex concentrates: a brief review. Eur J Anaesthesiol 2008; 25 (10): 784-9.

31. Fries D, Innerhofer P, Schobersberger W. Time for changing coagulation management in trauma-related massive bleeding. Curr Opin Anaesthesiol 2009; 22 (2): $267-74$

32. Sapsford W. The potential use of recombinant activated factor VII in trauma and surgery. Scand J Surg 2004; 93(1):17-23.
33. Scher C, Narine V, Chien D. Recombinant factor VIIa in trauma patients without coagulation disorders. Anesthesiol Clin 2010; 28(4):681-90.

34. Kenet G, Walden R, Eldad A, Martinowitz U. Treatment of traumatic bleeding with recombinant factor VIIa. Lancet. 1999; 354(9193):1879.

35. Perkins JG, Schreiber MA, Wade CE, Holcomb JB. Early verus late recombinant factor VIIa in combat trauma patients requiring massive transfusion. J Trauma 2007; 62(5):1095-9.

36. Spinella PC, Perkins JG, McLaughlin DF, Niles SA, Grathwohl KW, Beekley AC, et al. The effect of recombinant activated Factor VII on mortality in combat-related casualties with severe trauma and massive transfusion. J Trauma 2008; 64 (2): 286-94.

37. Boffard KD, Riou B, Warren B, Choong Pl, Rizoli S, Rossaint R, et al. Recombinant factor VIIa as adjunctive therapy for bleeding control in severely injured trauma patients: two parallel randomized, placebo-controlled, double-blind clinical trials. J Trauma 2005; 59(1): 8-15.

38. Lin Y, Stanworth S, Birchall J, Doree C, Hyde C. Recombinant factor VII for the prevention and treatment of bleeding in patients without haemophilia. Cochrane Database Sys Rev 2011; 16(2): CD005011.

39. Davies E, Smith A, Mahoney P, Midwinter M. Activated recombinant factor seven in British Military Trauma: an audit of use against SGOPL guidelines. JR Army Med Corps 2010; 156(3):178

40. Hodgetts TJ, Kirkman E, Mahoney PF, Russell R, Thomas R, Midwinter $M$. UK defence medical services guidance for the use of recombinant factor VIIA (RFVIIA) in the deployed military setting. JR Army Med Corps 2007; 153(4): 307-9.

41. Koshy JC, Hollier LH. Review of primary fibrinolysis is integral in the pathogenesis of the acute coagulopathy of trauma. J Craniofac Sur 2011; 22(5): 1980 .

42. The CRASH2 Collaborators. Effects of tranexamic acid on death, vascular occlusive events, and blood transfusion in trauma patients with significant hemorrhage (CRASH2): a randomized, placebo-controlled trial. Lancet 2011; 376 (9734):23-32.

43. Morrison JJ, Dubose JJ, Rasmussen TE, Midwinter MJ. Military application of tranexamic acid in trauma emergency resuscitation (MATTERs) study. Arch Surg. En prensa 2011.

44. Dawes R, Thomas GO. Battlefield resuscitation. Curr Opin Crit Care 2009; 15(6):527-35.

45. Deal VT, McDowell D, Benson P, Iddins B, Gluck G, Griffay A. et al. Tactical combat casualty care February 2010, direct from the battlefield: TCCC lessons learned in Iraq and Afghanistan. J Spec Oper Med 2010:10(3); 77 119 .

46. Anónimo. Battlefield Advanced Trauma Life Support. J R Army Med Corps 2004; 150(1): 32-40

47. Inspección General de Sanidad. Manual de asistencia al paciente crítico de las Fuerzas Armadas. Madrid. Ministerio de Defensa; 2009.

48. Hodgetts TJ, Mahoney PF, Russell MQ, Byers M. ABC to $<\mathrm{C}>\mathrm{ABC}$ : redefining the military trauma paradigm. Emerg Med J 2006; 23 (10):745-6.

49. González Alonso V, Cuadra Madrid ME, Usero Pérez MC, Colmenar Jarillo G, Sánchez Gil MA. Control de la hemorragia externa en combate. Prehosp Emerg Care 2009; 2(4): 293-304

50. Pozza M. Controllo dell'emorragia massiva in ambiente extra ospedaliero. N\&A mensile italiano del soccorso. 2010; 209: 2-8.

51. Lawton G, Granville-Chapman J, Parker PJ. Novel haemostatic dressings. JR Army Med Corps 2009; 155(4): 309-14.

52. Alam HB, Burris D, DaCorta JA, Rhee P. Hemorrhage control in the battlefield: role of new hemostatic agent. Mil Med 2005; 170(1): 63-9.

53. Cooper BR. Intra-osseous access (EZ-IO) for resucitation: UK military combat experience. J R Army Med Corp 2007; 153 (4):314-6.

54. La sangre, en misiones de rescate, aumenta la tasa de supervivencia de los heridos. Revista Atenea. El observatorio. 20 diciembre de 2010. N ${ }^{\circ}$ 157. 14-15.

55. Arnaud F, Tomori T, Carr W, McKeague A, Teranishi K, Prusaczyk K, et al. Exothermic reaction in zeolite hemostatic dressings: QuickClot ACS and ACS+. Annals of Biomedical Engineering 2008; 36 (10):1708-13.

56. Blackbourne LH. Combat damage control surgery. Crit Care Med 2008; 36(7): 304-10.

57. Sambasivan CN, Underwood SJ, Cho SD, Kiraly LN, Hamilton GJ, Kofoed $\mathrm{JT}$, et al. Comparison of abdominal damage control surgery in combat versus civilian trauma. J Trauma 2010; 69 (1): S168-74.

58. AJP-4.10(A) Allied Joint Medical Support Doctrine. NATO. 


\section{R. Navarro Suay, et al.}

59. Midwinter MJ, Brooks AJ. Damage control surgery: concepts. En: Brooks A. Ryan's ballistic trauma. London: Springer; 2011. p. 317-22.

60. Mathieu L, Bazile F, Barthélémy R, Duhamel P, Rigal S. Damage control orthopaedics in the contex of battlefield injuries: The use of temporally external fixation on combat trauma soldier. Orthop Traumatol Surg Res. En prensa 2011.

61. Brooks AJ, Cotton BA. Damage control resuscitation. En: Brooks A. Ryan's ballistic trauma. London: Springer; 2011. p. 251-62.

62. McFarland CC. Anesthesia for the ballistic casualty. En: Brooks A. Ryan's ballistic trauma. London: Springer; 2011. p. 287-98.

63. Niles SE, McLaughlin DF, Perkins JG, Wade CE, Li Y, Spinella PC, et al. Increased mortality associated with the early coagulopathy of trauma in combat casualties. J Trauma 2008; 64 (6): 1459-65.

64. Beekley AC, Starnes BW, Sebesta JA. Lessons learned from modern military surgery [review]. Surg Clin North Am 2007; 87 (1):157-84.

65. Arthurs Z, Cuadrado D, Beekley A, Grathwohl K, Perkins J, Rush R, et al The impact of hypothermia on trauma care at the 31 st combat support hospital. Am J Surg 2006;191(5):610-4.

66. Nesbitt M, Allen P, Beekley A, Butler F, Eastridge B, Blackbourne L. Current practice of thermoregulation during the transport of combat wounded. J Trauma 2010; 69 (1): S162-7.
67. Allen PB, Salyer SW, Dubick MA, Holcomb JB, Blackbourne LH. Preventing hypothermia: comparison of current devices used by the US Army in an in vitro warmed fluid model. J Trauma 2010; 69 (1): S154-61.

68. Collins JA, Simmons RL, James PM, Bredenberg CE, Anderson RW, Heisterkamp CA. The acid-base staqtus of seriously wounded combat casualties. Before treatment. Ann Surg 1970; 171(4):595-608.

69. Kashuk JL, Moore EE, Sawyer M, Le T, Johnson J, Biffl WL, et al. Postinjury coagulopathy management: goal directed resuscitation via POC thrombelastography. Ann Surg 2010; 251(4):604-14.

70. Craig J, Aguiar-Ibanez R, Bhattacharya S, Downie S, Duffy S, Kohli H Health Technology Assessment Report 11: the clinical and cost effectiveness of thromboelastography/thromboelastometry. Jun 2008. Disponible en: www. nhshealthquality.org. [citado 30 nov 2011]

71. Martinaud CH, Asencio Y, Ausset S, Sailliol A. Place de la thrombo-élastographie dans la prise en charge des hémorragies massives. Médecine et armées 2011; 39 (3): 269-70

72. Doran CM, Woolley T, Midwinter MJ. Feasibility of using rotational thromboelastometry to assess coagulation status of combat casualties in a deployed setting. J Trauma 2010; 69 (1):S40-8 